\title{
Systematic elucidation of the pharmacological mechanisms of Rhynchophylline for treating epilepsy via network pharmacology
}

\author{
Hua Geng ${ }^{1}$, Xuqin Chen ${ }^{2^{*}}$ and Chengzhong Wang ${ }^{1 *}$
}

\begin{abstract}
Abstact
Background: Epilepsy, one of the most common neurological disorders, affects over 70 million people worldwide. Rhynchophylline displays a wide variety of pharmacologic actives. However, the pharmacologic effects of rhynchophylline and its mechanisms against epilepsy have not been systematically elucidated.

Methods: The oral bioavailability and druglikeness of rhynchophylline were evaluated using the Traditional Chinese Medicine Systems Pharmacology Database. Rhynchophylline target genes to treat epilepsy were identified using PharmMapper, SwissTargetPrediction and DrugBank databases integration. Protein-protein interaction analysis was carried out by utilizing the GeneMANIA database. WebGestalt was employed to perform Gene ontology and Kyoto Encyclopedia of Genes and Genomes pathway enrichment analyses. The drug-disease-target-Gene Ontologypathway network was constructed using Cytoscape.

Results: The oral bioavailability and druglikeness of rhynchophylline were calculated to be $41.82 \%$ and 0.57 , respectively. A total of 20 rhynchophylline target genes related to epilepsy were chosen. Among the 20 genes and their interacting genes, $54.00 \%$ shared protein domains and $16.61 \%$ displayed co-expression characteristics. Gene ontology, Kyoto Encyclopedia of Genes and Genomes and network analyses illustrate that these targets were significantly enriched in regulation of sensory perception, morphine addiction, neuroactive ligand-receptor interaction and other pathways or biological processes.
\end{abstract}

Conclusion: In short, rhynchophylline targets multiple genes or proteins, biological processes and pathways. It shapes a multiple-layer network that exerts systematic pharmacologic activities on epilepsy.

Keywords: Rhynchophylline, Epilepsy, Drug targets, Gene ontology and Kyoto encyclopedia of genes and genomes, Network pharmacology

\footnotetext{
*Correspondence: xuqinlili@163.com; wangchengzhong2018@163.com

${ }^{2}$ Pediatric neurology department, Children's Hospital Affiliated To Suzhou

University, No. 50 Donghuan road, Suzhou 215000, Jiangsu Province, China 'Department of Pediatrics, Maternal and Child Health Hospital of Yancheng, No. 21 Century avenue, Yancheng 224002, Jiangsu Province, China
}

(c) The Author(s). 2021 Open Access This article is licensed under a Creative Commons Attribution 4.0 International License, which permits use, sharing, adaptation, distribution and reproduction in any medium or format, as long as you give appropriate credit to the original author(s) and the source, provide a link to the Creative Commons licence, and indicate if changes were made. The images or other third party material in this article are included in the article's Creative Commons licence, unless indicated otherwise in a credit line to the material. If material is not included in the article's Creative Commons licence and your intended use is not permitted by statutory regulation or exceeds the permitted use, you will need to obtain permission directly from the copyright holder. To view a copy of this licence, visit http://creativecommons.org/licenses/by/4.0/ The Creative Commons Public Domain Dedication waiver (http://creativecommons.org/publicdomain/zero/1.0/) applies to the data made available in this article, unless otherwise stated in a credit line to the data. 


\section{Background}

Epilepsy is a chronic nervous system disorder that exhibits abnormal brain activities, periods of unusual behavior or seizures, and sensations [1]. It affects over 70 million people of all ages worldwide. About $80 \%$ of people with epilepsy live in low or middle income countries. Nearly $70 \%$ of people with epilepsy can live seizure free if diagnosed and treated properly. However, over $75 \%$ of individuals are untreated, or even stigmatized in poor or developing countries [2].

Traditional Chinese medicine (TCM) contains the most abundant bioactive compounds or pharmaceutic ingredients for drug development. Uncaria rhynchophylla (U. rhynchophylla), for example, is a vital TCM that has been widely utilized in many Asian countries for centuries [3]. It is also known as Gou Teng in China, and found throughout Southern of China, including regions such as, Guangxi, Sicuan, Yunnan, and Fujian [4]. $U$. rhynchophylla belongs to the Rubiaceae family [4] and exerts a wide variety of pharmacologic activities, like anti-inflammatory effects and neurotransmitter regulation [3].

Attention has been closely paid to $U$. rhynchophylla due to its potential role in the prevention and treatment of a wide variety of diseases, especially for epilepsy [3]. One of the most abundant active pharmacological components of $U$. rhynchophylla is rhynchophylline (Rhy, Fig. 1b), which composes $28-50 \%$ of the total alkaloid content in $U$. rhynchophylla $[5,6]$. These results indicate Rhy can be utilized to treat epilepsy.

However, the potential molecular functions and involved pathways that Rhy induces have not yet been studied. Network pharmacology provides a novel avenue to elucidate the molecular mechanisms behind TCM through pharmacokinetic evaluation, target prediction and network construction. This is an emerging approach for drug discovery based on poly-pharmacology and systems biology, and has been successfully utilized to unveil the multi-targets and synergistic effects of TCM. For instance, Chen et al. have systematically investigated the mechanisms behind Salvianolic Acid A (SAA) via network pharmacology [7]. Application of network pharmacology approaches to predict the potential drug targets and unveil the latent mechanism will save time and effort. More importantly, a network pharmacology based approach facilitates the progress of drug development. Here, we elucidated systematically the pharmacologic effects of Rhy for the treatment of epilepsy using computational approaches in present study. A workflow of the analysis processes for Rhy targets identification and mechanism investigation is shown in Fig. 1a.

\section{Methods}

\section{Evaluation of oral bioavailability and drug likeness}

The Traditional Chinese Medicine Systems Pharmacology database (TCMSP, http://lsp.nwu.edu.cn/tcmsp. php), is a systematic pharmacology resource [8] that provides the absorption, distribution, metabolism and excretion (ADME) properties of TCMs or compounds, including information on oral bioavailability $(\mathrm{OB})$, druglikeness (DL), and blood-brain barrier (BBB) $[9,10]$.

Among the provided information, DL and $\mathrm{OB}$ are the most important properties of administered drugs, since they aid in assessing the effects of drug distribution in the circulatory system, and how drug like compounds are in relation to factors like

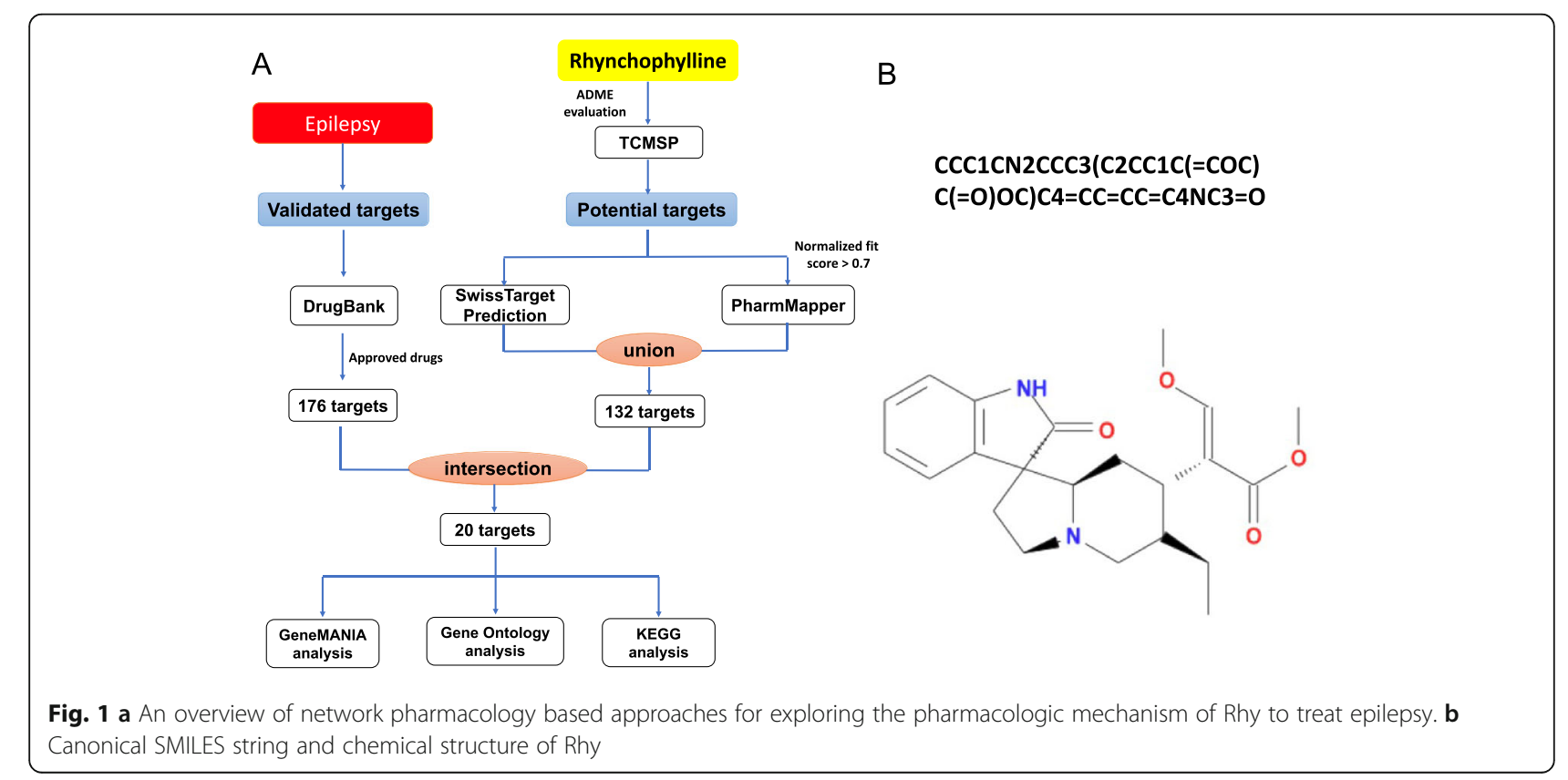


bioavailability. In the TCMSP database, DL was evaluated based on molecular descriptors and Tanimoto coefficients, and OB was calculated using OBioavail1.1 based on an in-house model [8,9].

Here, "rhynchophylline" was put into the TCMSP database and the pharmacokinetics properties of it were evaluated at the molecular level.

\section{Identification of targets via integrated database analyses} Putative targets of Rhy were predicted by using PharmMapper [11] and SwissTargetPrediction [12], while the validated epilepsy therapeutic targets were collected from the DrugBank [13] database. Overlaps between the predicted targets of Rhy and validated epilepsy therapeutic targets were considered as drug targets of Rhy for treating epilepsy.

PharmMapper (http://www.lilab-ecust.cn/pharmmapper/) is a reverse screening web tool was used to predict putative targets prediction of small molecules [11]. The Mol2 format file of Rhy downloaded from TCMSP was uploaded to the PharmMapper database. Human Protein targets only was selected along with other default arguments. Targets with a normalized fit score greater than 0.7 were selected for further investigation.

SwissTargetPrediction is a web tool used to identify the most possible target genes of small molecules, based on similarity principles via reverse docking [12]. A canonical SMILES string of Rhy was obtained from the PubChem database and uploaded to the SwissTargetPrediction server with all default parameters.

DrugBank is an online cheminformatics and freely accessible database that contains detailed information on drugs with comprehensive drug targets information [13]. The word "epilepsy" was put into DrugBank and only targets of drug approved by the Food and Drug Administration (FDA) acting on epilepsy were collected.

\section{GeneMANIA analysis}

GeneMANIA is an user-friendly and flexible online tool for analyzing gene sets, generating hypotheses concerning gene functions, and prioritizing genes for functional assays [14].

GeneMANIA identifies the most related genes based on correlations of curated genomics and proteomics information. After selecting Homo sapiens from the optional organisms, the target genes we identified were entered into the search box, and the results were further collected.

\section{Gene function and pathway enrichment analyses}

The Web-based gene set analysis toolkit (WebGestalt) was employed to explore systematically the functions and pathways of drug targets [15]. The gene list was entered into the WebGestalt web server using the overrepresentation enrichment analysis (ORA) approach with Gene Ontology (GO) and Kyoto Encyclopedia of Genes and Genomes pathway (KEGG) pathway databases and other default parameters. A false discovery rate (FDR) below 0.05 was considered as statistically significant.

GO analysis is extensively employed to annotate gene functions including molecular functions, and cellular components and biological processes, [16]. KEGG is used to systematically investigate pathway information for genes [17].

\section{Network construction}

To comprehensively understand the complex associations among the drug, disease, drug targets and associated biological process/pathways, Cytoscape (v 3.8.2) was employed to construct and analyze the multiplelayer network. Topological analysis was carried out using the network analyzer module in Cytoscape. Three topological properties including closeness, betweenness and degree were applied to evaluate the central properties of the nodes in the network.

\section{Results}

\section{Pharmacokinetics properties of Rhy}

TCMSP provides detailed information for key ADMErelated activities like human $\mathrm{OB}, \mathrm{DL}$, and $\mathrm{BBB}$. Using the TCMSP, AMDE-related properties of Rhy were deeply and fully studied. Of note, the OB and DL of Rhy were calculated as $41.82 \%$ and 0.57 , respectively (Fig. $1 \mathrm{~b}$ and Table 1), which were more higher than the TCMSP recommended thresholds ( $\mathrm{OB} \geq 30 \%, \mathrm{DL} \geq 0.18)$ [8]. These findings indicated that Rhy was chemically suitable for drug development.

\section{Drug targets of Rhy for treating epilepsy}

Putative targets of Rhy were predicted using PharmMapper [11] and SwissTargetPrediction [12]. After removing duplicates, 132 putative Rhy targets were obtained by combining information between two databases. A total of 176 validated therapeutic targets for epilepsy were acquired from the DrugBank database (Table S1). To understand the potential mechanisms of Rhy on epilepsy, those two parts targets were intersected and 20 genes in total were obtained finally. These 20 identified target genes were used for further analyses (Table 2).

\section{GeneMANIA analysis}

Protein-protein interaction (PPI) analysis was carried out using GeneMANIA to investigate the complex functional interaction network. Among the 20 target genes and their interacting genes, $54.00 \%$ shared protein domain, $17.73 \%$ were predicted, and $16.61 \%$ displayed co-expression characteristics. Other results, including co-localization and 
Table 1 Pharmacological and molecular properties of Rhy

\begin{tabular}{lllllllllll}
\hline ID & MW & AlogP & Hdom & Hacc & OB(\%) & Caco2 & BBB & DL & FSAF & HL \\
\hline Rhy & 384.2 & 1.92 & 1 & 6 & 41.82 & 0.47 & 0.38 & 0.57 & 0.23 & 13.2 \\
\hline
\end{tabular}

physical interactions, are demonstrated in Fig. 2. Altogether, these data suggested that Rhy may exert pharmacological functions via affecting multiple genes with shared protein domain or co-expression properties.

\section{GO and KEGG pathway analyses}

For further understanding the biological functions and involved pathways of the 20 identified target genes, GO and KEGG enrichment analyses were carried out using WebGestalt. The top seven engaged functions included biological regulation (20/20), cell communication (17/ $20)$, metabolic process (17/20), response to stimulus (17/ 20 ), and membrane $(17 / 20)$ (Fig. 3). These GO terms were related to anti-inflammation, in particular antiinflammation associated with epilepsy.

Moreover, we found that the 20 target genes were significantly related to regulation of sensory perception, regulation of transport, cGMP catabolic process, regulation of system process and iron transport, etc. (Fig. 4a and Table S2). As for the pathway analysis, genes enriched in the 8 KEGG pathways with significant FDRs included genes involved in morphine addiction, neuroactive ligand- receptor interaction, renin secretion, and the cGMP-PKG signaling pathway (Fig. 4b and Table S2).

\section{Network analysis}

Based on target identification, GO and pathway enrichment analyses, an entire drug, disease, targets and involved GO terms and KEGG pathways network was constructed using Cytoscape (v 3.8.2). This five-layer network contained 40 nodes and 141 edges (Fig. 5), suggesting multiple targets and multiple effects characteristics of Rhy for treating epilepsy,. Among these nodes, 20 were target gene nodes, 10 were GO function nodes and 8 were KEGG pathway nodes. The yellow and red oblong, blue circles, green inverted triangles, and purple triangles represent rhynchophylline, epilepsy, target genes and related GO biological processes and KEGG pathways, respectively. The degree of the 20 bioactive target genes in the network were displayed in Table 3 . Among these target genes, 5 had a high degree (degree $\geq 10$ ), these included ADOR1 (degree =14), OPRM1 (degree $=12)$, OPRD1 $($ degree $=11)$, PDE2A $\quad($ degree $=10)$ and OPRK1 (degree $=10)$.

Table 2 Target genes of Rhy for treating epilepsy

\begin{tabular}{|c|c|c|c|}
\hline No. & Gene ID & Gene Symbol & Target Name \\
\hline 1 & 134 & ADORA1 & adenosine A1 receptor \\
\hline 2 & 153 & ADRB1 & adrenoceptor beta 1 \\
\hline 3 & 472 & ATM & ATM serine/threonine kinase \\
\hline 4 & 771 & CA12 & carbonic anhydrase 12 \\
\hline 5 & 760 & CA2 & carbonic anhydrase 2 \\
\hline 6 & 8913 & CACNA1G & calcium voltage-gated channel subunit alpha1 G \\
\hline 7 & 1137 & CHRNA4 & cholinergic receptor nicotinic alpha 4 subunit \\
\hline 8 & 4128 & MAOA & monoamine oxidase A \\
\hline 9 & 4129 & MAOB & monoamine oxidase B \\
\hline 10 & 4985 & OPRD1 & opioid receptor delta 1 \\
\hline 11 & 4986 & OPRK1 & opioid receptor kappa 1 \\
\hline 12 & 4988 & OPRM1 & opioid receptor mu 1 \\
\hline 13 & 10,846 & PDE10A & phosphodiesterase 10A \\
\hline 14 & 5137 & PDE1C & phosphodiesterase $1 \mathrm{C}$ \\
\hline 15 & 5138 & PDE2A & phosphodiesterase 2A \\
\hline 16 & 8622 & PDE8B & phosphodiesterase 8B \\
\hline 17 & 5152 & PDE9A & phosphodiesterase 9A \\
\hline 18 & 5290 & PIK3CA & phosphatidylinositol-4,5-bisphosphate 3-kinase catalytic subunit alpha \\
\hline 19 & 6331 & SCN5A & sodium voltage-gated channel alpha subunit 5 \\
\hline 20 & 6532 & SLC6A4 & solute carrier family 6 member 4 \\
\hline
\end{tabular}




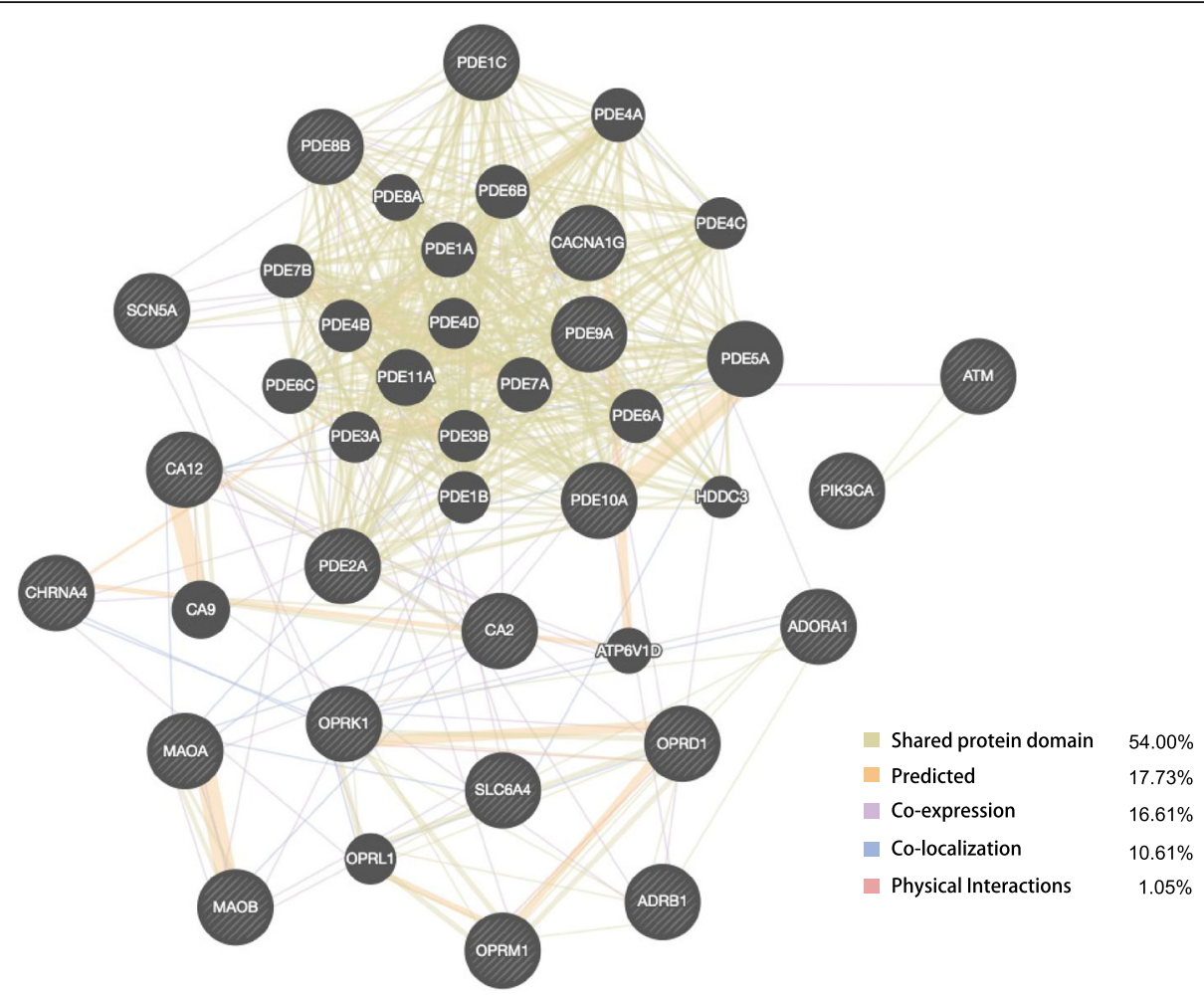

Fig. 2 PPI network of the Rhy target genes. Black nodes indicate target genes, while the edge colors represent different interactions. Genes in black circles were the query terms and these in grey circle illustrate genes related to the query genes

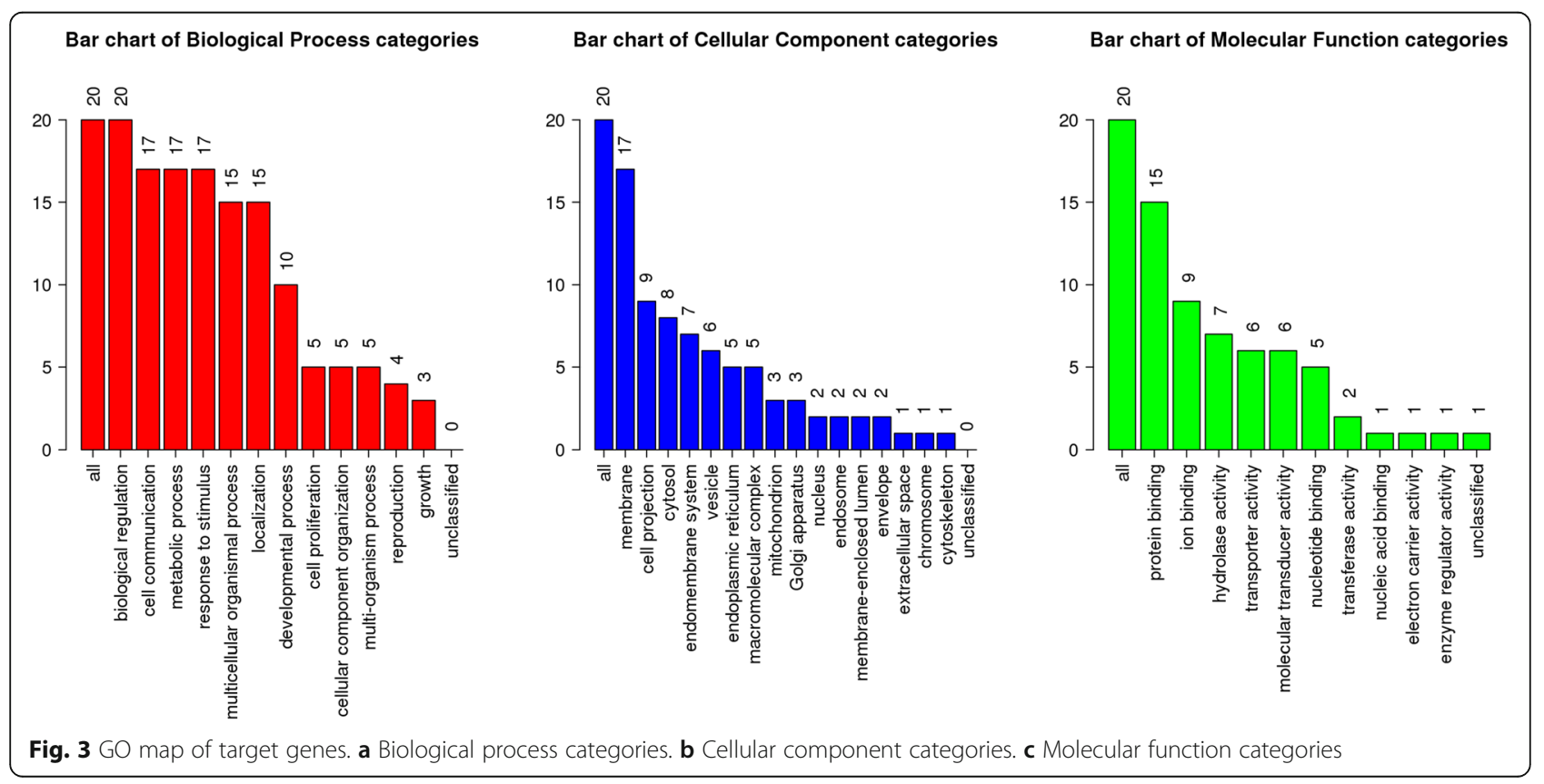


A

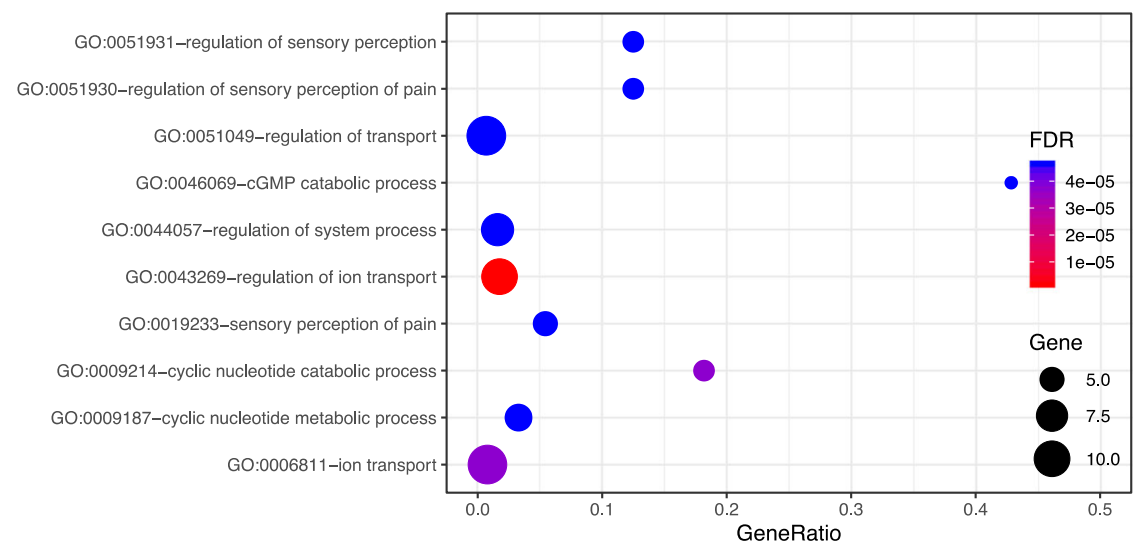

B

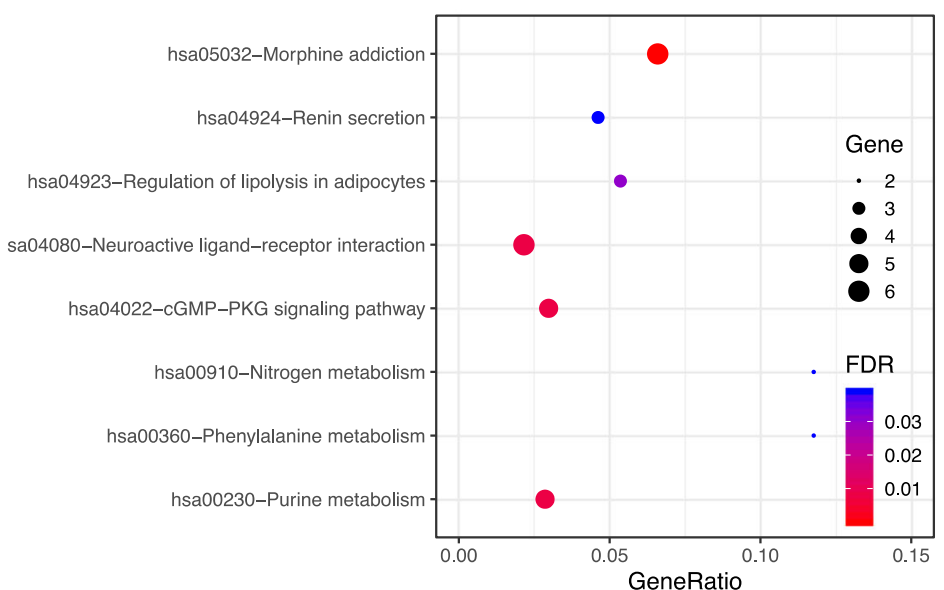

Fig. 4 Biological process (a) and pathway (b) enrichment analyses of target genes. X-axis represents gene ratio (gene count/gene size) while yaxis illustrates the enriched terms with ID and name

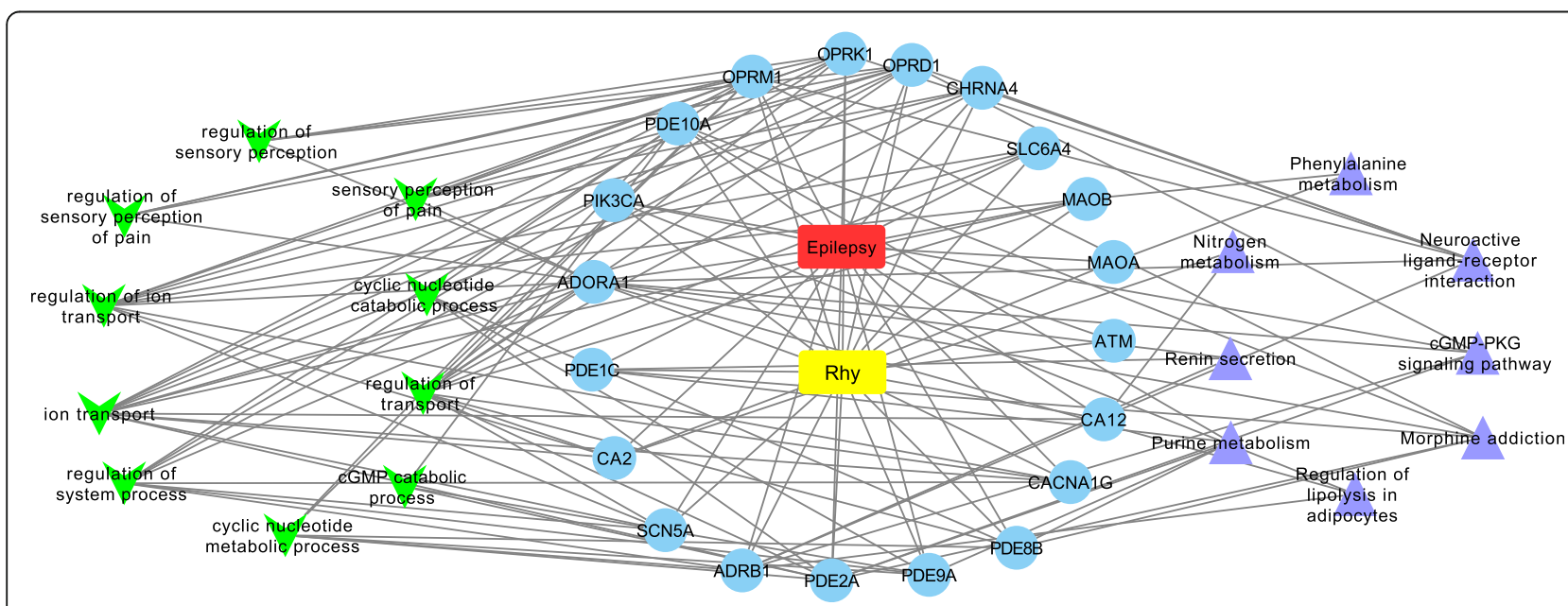

Fig. 5 Drug-disease-target-GO-pathway network. The yellow and red oblong, blue circles, green inverted triangles, and purple triangles represent rhynchophylline, epilepsy, target genes and related GO biological processes and KEGG pathways, respectively 
Table 3 The topological parameters of target genes sorted by degree

\begin{tabular}{llll}
\hline Target genes & Degree & Closeness & Betweenness \\
\hline ADORA1 & 14 & 0.557 & 0.119 \\
OPRM1 & 12 & 0.527 & 0.069 \\
OPRD1 & 11 & 0.513 & 0.051 \\
PDE2A & 10 & 0.5 & 0.075 \\
OPRK1 & 10 & 0.5 & 0.039 \\
ADRB1 & 8 & 0.476 & 0.043 \\
PDE9A & 7 & 0.464 & 0.038 \\
PDE10A & 7 & 0.464 & 0.033 \\
PDE8B & 7 & 0.464 & 0.029 \\
CHRNA4 & 7 & 0.464 & 0.016 \\
MAOB & 6 & 0.453 & 0.037 \\
CA2 & 6 & 0.453 & 0.034 \\
CACNA1G & 6 & 0.453 & 0.008 \\
SCN5A & 6 & 0.453 & 0.008 \\
PDE1C & 5 & 0.443 & 0.019 \\
SLC6A4 & 5 & 0.443 & 0.004 \\
CA12 & 4 & 0.433 & 0.021 \\
PIK3CA & 4 & 0.433 & 0.011 \\
MAOA & 3 & 0.424 & 0.017 \\
ATM & 2 & 0.415 & 0 \\
\hline
\end{tabular}

\section{Discussion}

Epilepsy, one of the most common serious brain diseases, affects 70 million people worldwide [2]. However, $30-40 \%$ of epileptic patients are resistant to western medicine and affected by side effects of these drugs [18]. TCM or natural products have been utilized widely to prevent and treat numerous diseases due to their high efficiency, small side effects and lack of drug resistance [19, 20]. Therefore, the development of active compounds extracted from TCM on drug design and discovery process should be prioritized urgently [21-24]. Here, we performed network pharmacology based analyses to screen the putative active compound and hidden mechanism of $U$. rhynchophylla acting on epilepsy.

Based on ADME properties, Rhy $(\mathrm{OB}=41.82 \%, \mathrm{DL}=$ 0.57 ) was screened as a potential bioactive compound for drug development. A total of 20 candidate targets of Rhy against epilepsy were identified, reflecting the multiple targets paradigm of TCM, GeneMANIA analysis suggested that these target genes and their associated genes share the same protein domain and have identical or similar functions.

To investigate the synergistic mechanisms of Rhy for the treatment of epilepsy, we carried out GO and KEGG pathway enrichment analysis. We identified an anti- inflammatory role of Rhy acting on epilepsy. Hsu et al. reported that seizures result in the inflammation of the central nervous system [25]. Moreover, we found that Rhy targets were closely related to biological processes, such as regulation of sensory perception, cGMP catabolic process, and regulation of system process. These findings were confirmed by previous experimental studies or reviews. For example, Campen et al. reported altered sensory sensitivity was associated with epilepsy susceptibility in childhood [26]. Nieoczym et al. revealed the involvement of the cGMP pathway in seizure processes [27]. SedighSarvestani et al. stated that regulatory systems, including the consciousness system and autonomic nervous system, play a vital role in the epileptic networks [28].

In addition, we discovered that the 20 Rhy targets acting on epilepsy were significantly enriched in morphine addiction, neuroactive ligand-receptor interaction, cGMP-PKG signaling and other pathways. Saboory et al. studied the mechanisms of morphine enhancement of spontaneous seizure activity and found that morphine at a low concentration (10 microM) depresses seizure activity [29]. Krasniqi et al. recently clarified the mechanism of renin in brain induced neuropathology like epilepsy, and discovered renin inhibitors may have therapeutic applications for epilepsy treatment [30]. Liu et al. reported that abnormal neuroactive ligand-receptor interactions enhance the susceptibility to epileptic seizures [31]. Moreover, Risley et al. proposed that the cGMP-PKG signaling pathway was a hidden new target for studying epilepsy mechanisms [32]. These finding closely coincide with the results obtained through GO and KEGG analyses.

The multiple-layer network illustrated that Rhy has multiple targets to exert multiple pharmacologic effects, reflecting multiple targets, multiple effects and complex disease. In total, 5 of 20 targets displayed a degree larger than 10 in the network, including ADOR1, OPRM1, OPRD1, PDE2A and OPRK1. Some of them have been verified by animal experiments or clinical trials. Kasai et al. reported that the OPRM1 polymorphism was associated with epilepsy susceptibility [33]. Similarly, Doummar et al. also reported PDE2A variants, identified by whole-exome sequencing, caused cognitive impairment and occurred in epilepsy patients [34]. Ueda et al. revealed an upregulation of OPRK1 in a rat model of posttraumatic epilepsy [35]. There are no previous studies directly reporting associations between the other 2 key targets (ADOR1, OPRD1) and epilepsy.

Taken together, we propose that Rhy may be a promising compound that can be applied for further drug development in the treatment of epilepsy. Despite these findings, there were still some bias and limitations in this study owing to the databases utilized in analyses. Further studies are necessary to validate the acting mechanisms via pharmacokinetic tests. 


\section{Conclusion}

In conclusion, Rhy is a promising and valuable compound for the development of a safe and effective multitargeted anti-epilepsy therapy. This study sheds insight on the perspectives and challenges of Rhy research and clinical applications acting on epilepsy in the future.

\section{Supplementary Information}

The online version contains supplementary material available at https:/doi. org/10.1186/s12906-020-03178-x

Additional file 1. Detail information of the putative targets of Rhy and validated therapeutic targets on epilepsy.

Additional file 2. Detail information of GO and KEGG enrichment analyses.

\section{Abbreviations}

TCMSP: Traditional Chinese Medicine Systems Pharmacology Database; GO: Gene Ontology; TCM: Traditional Chinese Medicine; U. rhynchophylla: Uncaria rhynchophylla; Rhy: rhynchophylline: ADME: Absorption, Distribution, Metabolism and Excretion; DL: Drug Likeness; OB: Oral Bioavailability; BBB: Blood-Brain Barrier; FDA: Food and Drug Administration; PPI: Protein-protein interaction; WebGestalt: Web-based Gene set analysis toolkit; ORA: Overrepresentation Enrichment Analysis; FDR: False discovery rate; KEGG: Kyoto Encyclopedia of Genes and Genomes pathway

\section{Acknowledgments}

We would like to thank Hongguo Shao for manuscript review and English language editing help in this study.

\section{Authors' contributions}

WCZ and CXQ conceived the idea of this article and supervised the research. $\mathrm{GH}$ performed the research, analyzed the data and wrote the manuscript. WCZ and CXQ participated in revising the data and improving manuscript writing. All authors have read and approved the final version of the manuscript.

\section{Funding}

This work was financially supported by the grants from Jiangsu province maternal and child health research project fund (No. F201749).

\section{Availability of data and materials}

All main data has been presented in the form of tables in additional file. Other data used to support the findings of this study are available from the corresponding author upon request.

Ethics approval and consent to participate

Not applicable.

\section{Consent for publication}

Not applicable.

\section{Competing interests}

The authors declare no conflicts of interest in this work.

Received: 16 December 2019 Accepted: 7 December 2020 Published online: 06 January 2021

\section{References}

1. Fisher RS, et al. ILAE official report: a practical clinical definition of epilepsy Epilepsia. 2014;55(4):475-82.

2. Thijs RD, et al. Epilepsy in adults. Lancet. 2019;393(10172):689-701.

3. Yang $W$, et al. Uncaria rhynchophylla and its major constituents on central nervous system: a review on their pharmacological actions. Curr Vasc Pharmacol. 2020;18(4):346-57.
4. Shi JS, et al. Pharmacological actions of Uncaria alkaloids, rhynchophylline and isorhynchophylline. Acta Pharmacol Sin. 2003;24(2):97-101.

5. Phillipson JD, Hemingway SR. Chromatographic and spectroscopic methods for the identification of alkaloids from herbarium samples of the genus Uncaria. J Chromatogr. 1975;105(1):163-78.

6. Yamanaka $E$, et al. Studies of plants containing indole alkaloids. IX. Quantitative analysis of tertiary alkaloids in various parts of Uncaria rhynchophylla MIQ. Yakugaku Zasshi. 1983;103(10):1028-33.

7. Chen SJ, Cui MC. Systematic understanding of the mechanism of salvianolic acid a via computational target fishing. Molecules. 201717;22(4):644-53.

8. Ru J, et al. TCMSP: a database of systems pharmacology for drug discovery from herbal medicines. J Cheminform. 2014;6:13.

9. Pei T, et al. Systematic understanding the mechanisms of vitiligo pathogenesis and its treatment by Qubaibabuqi formula. J Ethnopharmacol. 2016:190:272-87.

10. Zhang $W$, et al. Systems pharmacology dissection of the integrated treatment for cardiovascular and gastrointestinal disorders by traditional Chinese medicine. Sci Rep. 2016;6:32400.

11. Wang X, et al. PharmMapper 2017 update: a web server for potential drug target identification with a comprehensive target pharmacophore database. Nucleic Acids Res. 2017:45(W1):W356-60.

12. Daina A, Michielin O, Zoete V. SwissTargetPrediction: updated data and new features for efficient prediction of protein targets of small molecules. Nucleic Acids Res. 2019;47(W1):W357-64.

13. Wishart DS, et al. DrugBank 5.0: A major update to the DrugBank database for 2018. Nucleic Acids Res. 2018:46(D1):D1074-82

14. Warde-Farley D, et al. The GeneMANIA prediction server: biological network integration for gene prioritization and predicting gene function. Nucleic Acids Res. 2010;38(Web Server issue):W214-20

15. Wang J, et al. WEB-based GEne SeT AnaLysis Toolkit (WebGestalt): update 2013. Nucleic Acids Res. 2013;41(Web Server issue):W77-83

16. The Gene Ontology, C. Expansion of the Gene Ontology knowledgebase and resources. Nucleic Acids Res. 2017;45(D1):D331-8.

17. Kanehisa M, et al. KEGG: new perspectives on genomes, pathways, diseases and drugs. Nucleic Acids Res. 2017;45(D1):D353-61.

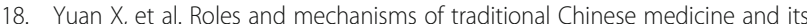
active ingredients in treating epilepsy. Zhongguo Zhong Yao Za Zhi. 2019; 44(1):9-18.

19. Li W, et al. Platycodin D exerts anti-tumor efficacy in $\mathrm{H} 22$ tumor-bearing mice via improving immune function and inducing apoptosis. J Toxicol Sci. 2016;41(3):417-28

20. Hossen MJ, et al. Codonopsis lanceolata: a review of its therapeutic potentials. Phytother Res. 2016;30(3):347-56.

21. van de Waterbeemd $\mathrm{H}$, Gifford E. ADMET in silico modelling: towards prediction paradise? Nat Rev Drug Discov. 2003:2(3):192-204.

22. Kou $Y$, et al. Synthesis of $14^{\prime}, 15^{\prime}$-dehydro-ritterazine $Y$ via reductive and oxidative functionalizations of hecogenin acetate. Steroids. 2013:78(2):304-11.

23. Kou Y, et al. Application of hypoiodite-mediated aminyl radical cyclization to synthesis of solasodine acetate. Steroids. 2012;77(11):1069-74.

24. Kou Y, Lee S. Unexpected opening of steroidal E-ring during hypoioditemediated oxidation. Tetrahedron Lett. 2013:54(31):4106-9.

25. Hsu HC, et al. Antiepileptic effect of Uncaria rhynchophylla and Rhynchophylline involved in the initiation of c-Jun N-terminal kinase phosphorylation of MAPK signal pathways in acute seizures of Kainic acidtreated rats. Evid Based Complement Alternat Med. 2013;2013:961289.

26. van Campen JS, et al. Sensory modulation disorders in childhood epilepsy. $J$ Neurodev Disord. 2015;7:34.

27. Nieoczym D, et al. Effect of sildenafil on the anticonvulsant action of classical and second-generation antiepileptic drugs in maximal electroshock-induced seizures in mice. Epilepsia. 2010;51(8):1552-9.

28. Sedigh-Sarvestani $M$, et al. Seizures and brain regulatory systems: consciousness, sleep, and autonomic systems. J Clin Neurophysiol. 2015; 32(3):188-93.

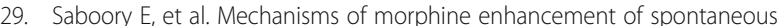
seizure activity. Anesth Analg. 2007;105(6):1729-35 table of contents.

30. Krasniqi S, Daci A. Role of the angiotensin pathway and its target therapy in epilepsy management. Int J Mol Sci. 2019:20(3):726-42.

31. Liu $X$, et al. New differentially expressed genes and differential DNA methylation underlying refractory epilepsy. Oncotarget. 2016;7(52):87402-16.

32. Risley $M G$, et al. egl-4 modulates electroconvulsive seizure duration in $C$. elegans. Invert Neurosci. 2018;18(2):8. 
33. Kasai S, Ikeda K. Pharmacogenomics of the human micro-opioid receptor. Pharmacogenomics. 2011;12(9):1305-20

34. Doummar D, et al. Biallelic PDE2A variants: a new cause of syndromic paroxysmal dyskinesia. Eur J Hum Genet. 2020;28(10):1403-13.

35. Ueda Y, et al. Hippocampal gene expression profiling in a rat model of posttraumatic epilepsy reveals temporal upregulation of lipid metabolismrelated genes. Neurochem Res. 2013;38(7):1399-406.

\section{Publisher's Note}

Springer Nature remains neutral with regard to jurisdictional claims in published maps and institutional affiliations.

Ready to submit your research? Choose BMC and benefit from:

- fast, convenient online submission

- thorough peer review by experienced researchers in your field

- rapid publication on acceptance

- support for research data, including large and complex data types

- gold Open Access which fosters wider collaboration and increased citations

- maximum visibility for your research: over $100 \mathrm{M}$ website views per year

At BMC, research is always in progress.

Learn more biomedcentral.com/submissions 